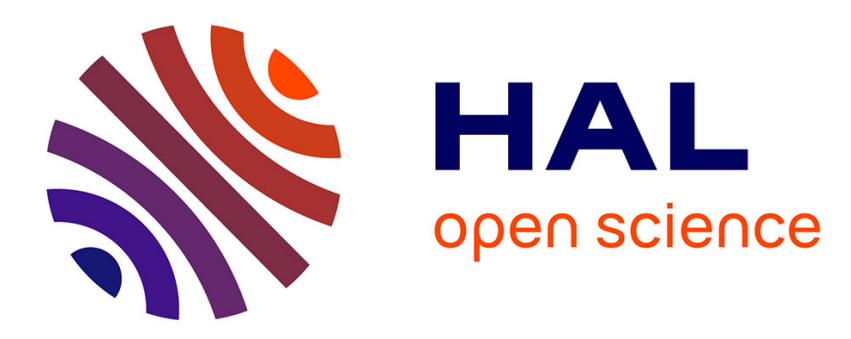

\title{
Mesure par thermographie infrarouge de la conductivité thermique longitudinale de plaques minces homogènes ou composites
}

\author{
C. Saint-Blanquet, R. Alnet
}

\section{- To cite this version:}

C. Saint-Blanquet, R. Alnet. Mesure par thermographie infrarouge de la conductivité thermique longitudinale de plaques minces homogènes ou composites. Revue de Physique Appliquée, 1985, 20 (8), pp.621-627. 10.1051/rphysap:01985002008062100 • jpa-00245377

\section{HAL Id: jpa-00245377 https://hal.science/jpa-00245377}

Submitted on 1 Jan 1985

HAL is a multi-disciplinary open access archive for the deposit and dissemination of scientific research documents, whether they are published or not. The documents may come from teaching and research institutions in France or abroad, or from public or private research centers.
L'archive ouverte pluridisciplinaire HAL, est destinée au dépôt et à la diffusion de documents scientifiques de niveau recherche, publiés ou non, émanant des établissements d'enseignement et de recherche français ou étrangers, des laboratoires publics ou privés. 


\title{
Mesure par thermographie infrarouge de la conductivité thermique longitudinale de plaques minces homogènes ou composites
}

\author{
C. Saint-Blanquet et R. Alnet \\ Laboratoire de Thermocinétique (*), Université de Nantes, 2, rue de la Houssinière, 44072 Nantes Cedex, France
}

(Reçu le 8 octobre 1984, révisé le 22 avril 1985, accepté le 29 avril 1985)

\begin{abstract}
Résumé. - Une méthode originale de mesure de la conductivité thermique des matériaux fait l'objet de cet article. Les champs de température relevés par thermographie infrarouge sont confrontés à une théorie de " barre semiinfinie » et donnent la valeur de la conductivité thermique. Par comparaison avec les méthodes classiques, on prouve la validité de notre travail pour les isolants et pour les conducteurs de la chaleur. On étudie alors des matériaux anisotropes, plaques constituées de couches isolantes et conductrices et on conclut sur les possibilités de cette méthode pour les substrats utilisés en électronique.
\end{abstract}

\begin{abstract}
This paper deals with an original method to measure the thermal conductivity. An infrared camera yields the temperature fields which are analysed by a " semi-infinite rod " theory; we thus obtain the thermal conductivity value. Otherwise, we have experimented the same material with a standard method and the excellent correlation between the two approaches proves the validity of our work for the heat insulators and good conductors. In order to check the validity of our method for electronics supports, we have also studied a bilayer plate with the same range of success.
\end{abstract}

On sait l'ìmportance que revêt, pour la prévision des phénomènes thermiques, une bonne connaissance de la conductivité thermique des matériaux. La détermination précise de ce paramètre implique sa mesure car les prévisions théoriques ne sont pas suffisamment sûres surtout lorsqu'on a affaire à des composites. Cette mesure s'effectue $[1,2]$ le plus souvent en régime permanent et la maîtrise des paramètres intervenant (méthode de la plaque gardée par exemple) impose aux échantillons une forme et des dimensions optimales qui parfois ne peuvent être réalisées. C'est le cas des plaques minces telles que les cartes utilisées pour les circuits en électronique lorsqu'on veut déterminer leur conductivité longitudinale. La méthode présentée dans cet article a été étudiée pour permettre commodément ce type de mesure.

Il s'agit d'une méthode originate :

- adaptée à des échantillons en forme de plaques minces;

- mesurant la conductivité dans le sens des dimensions étendues:

— où le relevé du champ de température est fait

(*) E.R.A. CNRS No 663 . par thermographie infrarouge ce qui supprime l'implantation de capteurs de température sur la plaque elle-même.

Le principe de la méthode est simple. La plaque est disposée verticalement dans l'air ambiant, la direction principale de conductivité à mesurer étant horizontale, on dissipe localement selon une ligne verticale un flux de chaleur connu qui diffuse dans la plaque et s'évacue vers l'ambiante par chacune de ses faces. On se place dans des conditions telles que cette plaque soit assimilable à une ailette plane; relevant en régime permanent le champ de température, on déduit la conductivité thermique.

\section{Etude théorique.}

Une plaque rectangulaire, verticale, d'épaisseur $e$ petite devant ses autres dimensions $(H, L)$ vérifie l'hypothèse de "barre " si le nombre de Biot $\left({ }^{1}\right)$ est inférieur à 0,1 .

( $\left.{ }^{1}\right)$ Nombre de Biot $: \frac{h e}{\lambda} ; h=$ coefficient d'échanges linéarisés, $e=$ épaisseur de la plaque, $\lambda=$ conductivité de la plaque. 
Un fil de thermocoax $\left({ }^{2}\right)$, inséré et collé dans une rainure au centre suivant la hauteur $H$, sert de source de chaleur. La plaque est suffisamment longue (dimen$\operatorname{sion} L$ ) et répond sur le plan théorique à l'hypothèse de milieu semi-infini.

Le courant électrique circulant dans le fil thermocoax impose un flux de chaleur qui se propage de manière symétrique dans la plaque. Ce flux de chaleur est évacué par convection naturelle et rayonnement thermique dans le milieu ambiant à température $\theta_{\mathrm{a}}$.

On tient compte des échanges par convection par une loi du type :

$$
\varphi_{\mathrm{c}}=k\left(\theta-\theta_{\mathrm{a}}\right)^{5 / 4}
$$

où $\theta$ est la température de la plaque à l'endroit considéré, $k$ un coefficient de proportionnalité dépendant de la hauteur $y$ à partir du bord inférieur $\left(^{3}\right)$ et $\varphi_{c}$ la densité de chaleur évacuée.

On tient compte des échanges par rayonnement par une loi du type :

$$
\varphi_{\mathrm{R}}=\varepsilon \sigma\left(\theta^{4}-\theta_{\mathrm{a}}^{4}\right)
$$

où $\varphi_{R}$ est la densité de flux de chaleur évacuée par rayonnement dans le milieu ambiant, $\sigma$ la constante de Stefan-Boltzmann, $\varepsilon$ l'émissivité de la surface de la plaque.

Un bilan de chaleur conduit aux systèmes d'équations :

$$
\left\{\begin{array}{l}
\frac{\mathrm{d}^{2} \theta}{\mathrm{d} x^{2}}-\frac{2 k_{1}}{\lambda e}\left(\theta-\theta_{\mathrm{a}}\right)^{5 / 4}-\frac{2 \varepsilon \sigma}{\lambda e}\left(\theta^{4}-\theta_{\mathrm{a}}^{4}\right)=0 \\
-\lambda\left(\frac{\mathrm{d} \theta}{\mathrm{d} x}\right)_{x=0}=\varphi_{0} \\
\theta(\infty)=\theta_{\mathrm{a}} .
\end{array}\right.
$$

Remarques :

- Dans l'équation (3), nous avons négligé le terme de conduction verticale $-\lambda \frac{\partial \theta}{\partial y}$ devant le terme de conduction horizontale $-\lambda \frac{\partial \theta}{\partial x}$. En fait, un gradient

(2) On prend du thermocoax de $5 / 10 \mathrm{~mm}$ de diamètre.

$\left({ }^{3}\right)$ Bien que les développements de couche limite soient différents pour le problème présenté, on rappelle les résultats couramment admis pour une plaque verticale à flux constant en convection naturelle.

La puissance 5/4 dans l'expression de $\varphi_{\mathrm{c}}$ suppose un nombre de Rayleigh $R a$ suffisamment faible :

$$
\begin{aligned}
& \varphi_{\mathrm{c}}(y)=\frac{0,401 \lambda_{\mathrm{f}}}{y}(R a)^{1 / 4}\left(\theta-\theta_{\mathrm{a}}\right) \text { si } 500<R a<2 \times 10^{7} \\
& \text { où } R a=G r \operatorname{Pr}, G r=\frac{\beta g \rho^{2} y^{3}}{\mu^{2}}\left(\theta-\theta_{\mathrm{a}}\right), \operatorname{Pr}=\frac{v}{a}=\frac{\mu}{\rho a} . \\
& \quad G r=\text { nombre de Grashoff; } \operatorname{Pr}=\text { nombre de Prandtl; } \\
& \begin{array}{l}
y=\text { hauteur considérée à partir du bord inférieur de } \\
\text { chauffage. }
\end{array}
\end{aligned}
$$

vertical de température existe-le coefficient $k_{1}$ dépend de $y$ - principalement au début du développement de la couche limite. Nous établirons les profils de température pour des ordonnées $y$ de l'ordre de 15 à $20 \mathrm{~cm}$ où le gradient vertical, expérimentalement, n'est pas significatif et l'analyse parfaitement justifiée.

- Dans l'équation (4), nous avons réparti uniformément en $x=0$ le flux de chaleur sur l'épaisseur $e$. Dans la réalisation pratique (insertion d'un fil thermocoax) nous avons une zone centrale avec un phénomène de constriction (Fig. 1). Nous y reviendrons lors du dépouillement expérimental.

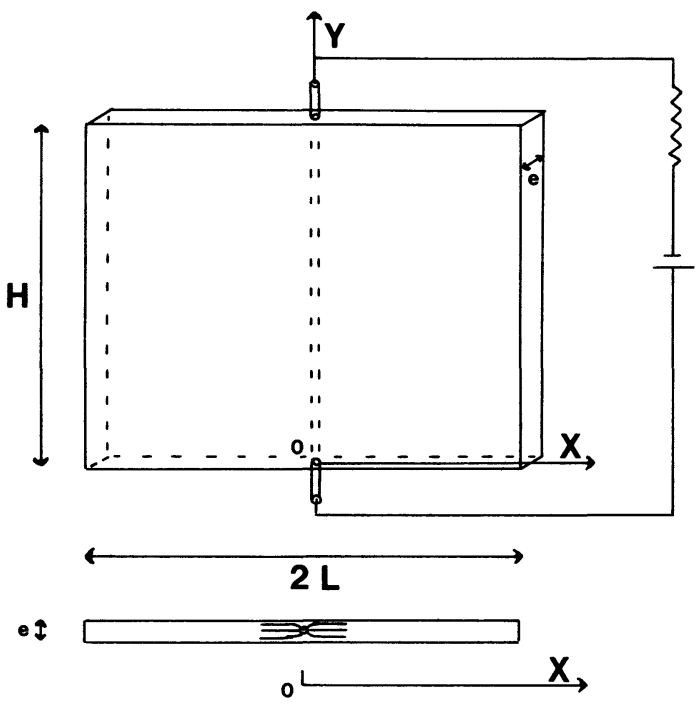

Fig. 1. - Schéma expérimental. [Experimental diagram.]

1.1 Solution analytiQue APPRochée. - Pour des températures $\theta$ comprises entre $323 \mathrm{~K}$ et $353 \mathrm{~K}$, pour $\theta_{\mathrm{a}}$ de l'ordre de $293 \mathrm{~K}$, le rapport $\frac{\theta^{4}-\theta_{\mathrm{a}}^{4}}{\left(\theta-\theta_{\mathrm{a}}\right)^{5 / 4}}=k_{2}$ est constant avec une stabilité supérieure à $1,5 \%$ autour de la valeur moyenne.

L'équation (3) devient (6), c'est-à-dire :

$$
\frac{\mathrm{d}^{2} \theta}{\mathrm{d} x^{2}}-K\left(\theta-\theta_{\mathrm{a}}\right)^{5 / 4}=0
$$

avec $K=\frac{2 k_{1}+2 \varepsilon \sigma k_{2}}{\lambda e}$

En posant $T=\theta-\theta_{\mathrm{a}}$ on est amené à intégrer le système :

$$
\left\{\begin{array}{l}
\frac{\mathrm{d}^{2} T}{\mathrm{~d} x^{2}}-K T^{5 / 4}=0 \\
-\lambda\left(\frac{\mathrm{d} T}{\mathrm{~d} x}\right)_{x=0}=\varphi_{0} \\
T(\infty)=0
\end{array}\right.
$$


dont la solution est :

$$
T^{-1 / 8}=\sqrt{\frac{K}{72}} x+T_{0}^{-1 / 8}
$$

qui conduit à :

$$
\lambda=\sqrt{\frac{9}{8 K}} T_{0}^{-9 / 8} \varphi_{0}
$$

1.2 SOlution NUMÉRIQUe. - Pour tester la validité de la solution analytique, il convenait de résoudre le système d'équations (3), (4), (5). La résolution numérique ne présente guère de difficultés. On utilise une méthode de relaxation, le critère de convergence étant classique, à savoir la somme des valeurs absolues des écarts à chaque maille entre la $i$-ième itération et la $(i-1)$-ième reste inférieure à un infiniment petit. Disposant de plus d'une solution approchée (solution analytique) pour initialiser les itérations, le schéma numérique est particulièrement simple, encore convient-il d'être attentif à l'équation (4).

Pour tester la solution analytique, nous calculons une valeur de $\lambda$ à partir de (10) et (11) et des profils obtenus par la résolution analytique. Nous comparons cette valeur de $\lambda$ à celle que nous avions entrée pour calculer les profils par résolution numérique.

Pour des températures maximales inférieures à $150^{\circ} \mathrm{C}$, les écarts ne sont pas significatifs et moindres que les erreurs expérimentales espérées. Pour des températures de $250^{\circ} \mathrm{C}$, on atteint des écarts de l'ordre de 3 à $4 \%$.

\section{Etude expérimentale.}

Les plaques sont systématiquement peintes, avec une peinture noire mate d'émissivité importante ( $\varepsilon$ supérieur à 0,9 ). (11).

La détermination de $\lambda$ se fait à partir de la formule

Pour les résultats que nous présentons, nous avons choisi une densité de flux $\varphi_{0}$ telle que la température maximale soit de l'ordre de $80^{\circ} \mathrm{C}$. Evidemment la connaissance exacte de $\varphi_{0}$ résulte de mesures électriques (tension, courant). La détermination des paramètres $K$ et $T_{0}$ est plus délicate. La caméra infrarouge relève directement sur écran de télévision un profil de température sur l'horizontale choisie. Le processus de transcription de ce "profil de température " en températures vraies, ainsi que quelques éléments nécessaires de compréhension du fonctionnement de la caméra infrarouge sont décrits en annexe.

Il convient de tenir compte du grandissement linéaire entre profil de température de la plaque et celui visualisé sur l'écran de télévision.

On mesure ce grandissement (Fig. 2) en traçant sur une horizontale voisine de celle de mesure deux points brillants (peinture d'argent) qui sont visibles sur la
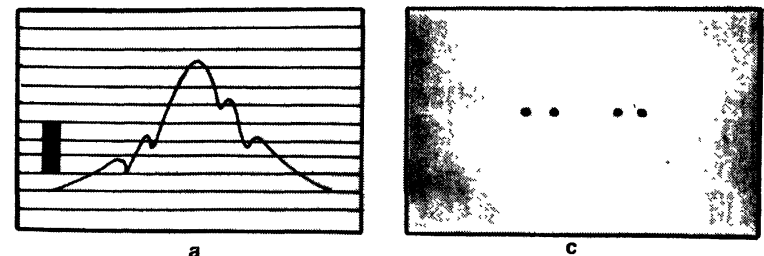
a : PROFIL THERMIQUE SUR LES
POINTS D'ARGENT

(THERMAL PROFILE ON THE SILVER POINTS)

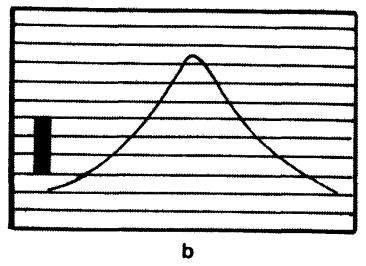

b : PROFIL THERMIQUE NORMAL (STANDARD THERMAL PROFILE)

c : THERMOGRAPHIE DE LA PLAQUE (PLATE'S THERMOGRAPHY)

Fig. 2. - Visualisation de la thermographie sur écran de télévision.

[Thermography's view on a television set.]

plaque et sur l'écran de télévision (l'émissivité de ces points étant plus faible, ils sortent plus foncés pour une même température).

2.1 DÉTERMINATION DE LA CONDUCTIVITÉ THERMIQUE LONGITUDINALE D'UN ISOLANT COMPOSITE (VERRE POLYIMIDE). $-H=0.258 \mathrm{~m} ; L=0,117 \mathrm{~m} ; e=1,6 \times$ $10^{-3} \mathrm{~m}$. Pour une densité de flux $\varphi_{0}=5650 \mathrm{~W} / \mathrm{m}^{2}$, nous avons représenté (Fig. 3) $T^{-1 / 8}$ en fonction de $x$.

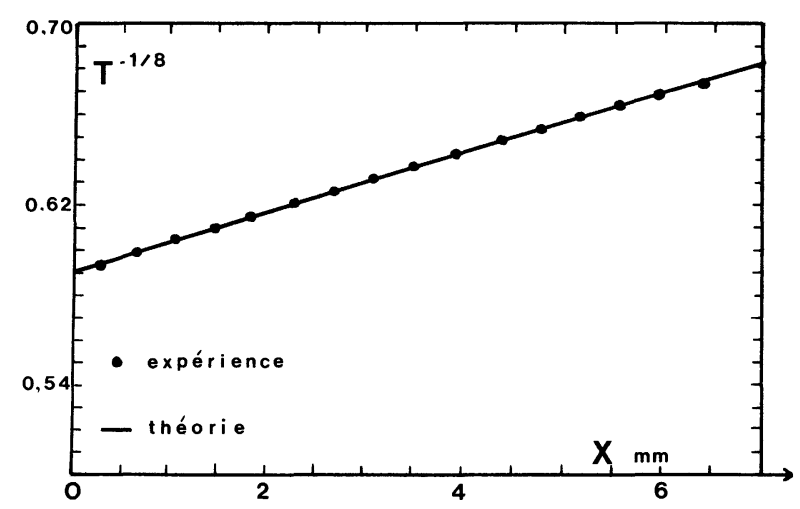

Fig. 3. - Thermogramme d'une plaque isolante.

[Heat insulator plate's thermogram.]

On constate que $T^{-1 / 8}$ évolue linéairement avec $x$, ce qui est en accord avec la solution analytique approchée, formule (10), sauf dans la zone centrale autour du thermocoax qui correspond à la zone perturbée par le phénomène de constriction. Par une méthode de régression linéaire, on calcule la pente $\sqrt{K / 72}$ et la valeur origine $T_{0}^{-1 / 8}$ et, par suite, la valeur de $\lambda$.

On obtient $K=11900 \mathrm{~m}^{-2} \cdot \mathrm{K}^{-1 / 4} ; T_{0}=68,4{ }^{\circ} \mathrm{C}$ et $\lambda=0,47$ (3) $\mathrm{W} / \mathrm{k} . \mathrm{m}$. 
Remarques :

- Nous avons mesuré la conductivité longitudinale du verre polyimide par une autre méthode [2] et trouvé $\lambda=0,48 \mathrm{~W} / \mathrm{m} . \mathrm{K}$.

- Pour justifier l'hypothèse de barre, nous avons calculé en linéarisant les pertes par convection et rayonnement le nombre de Biot $\left(\frac{h e}{\lambda} ; h\right.$ coefficient d'échanges linéarisées); nous avons trouvé 0,038 , ce qui est bien inférieur à 0,1 .

- Sur la figure 4 nous avons tracé la solution analytique $\theta=T+\theta_{\mathrm{a}}$ en fonction de $x$ et comparé avec les résultats expérimentaux, ceci pour bien mettre en évidence l'excellente corrélation et faire apparaître la zone perturbée.

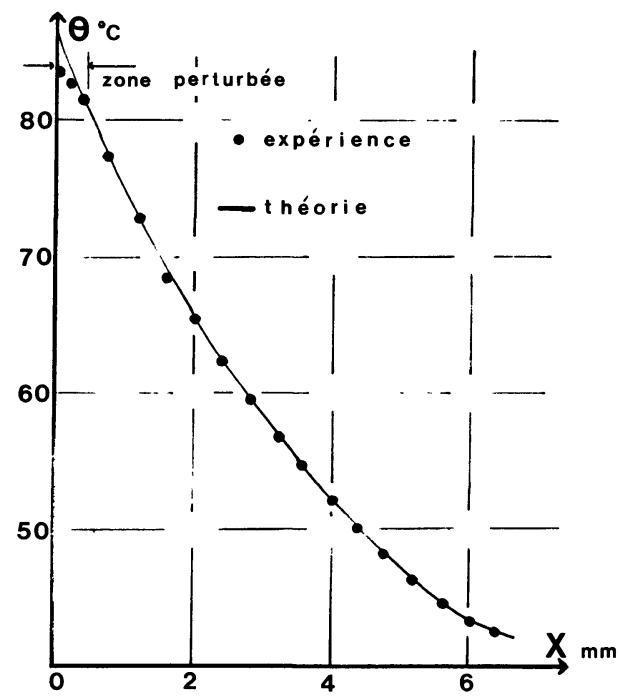

Fig. 4. - Thermogramme d'une plaque isolante. [Heat insulator plate's thermogram.]

2.2 DÉTERMINATION DE LA CONDUCTIVITÉ THERMIQUE D'UN CONDUCTEUR (FER A 42). $-H=0,250 \mathrm{~m}$; $L=0,200 \mathrm{~m} ; e=3 \times 10^{-3} \mathrm{~m}$. Pour une densité de flux de chaleur $\varphi_{0}=35690 \mathrm{~W} / \mathrm{m}^{2}$, la figure 5 représente $T^{-1 / 8}$ en fonction de $x$. L'évolution linéaire est en accord avec la formule (10).

On obtient $K=74,9 \mathrm{~m}^{-2} \cdot \mathrm{K}^{-1 / 4} ; T_{0}=61,1{ }^{\circ} \mathrm{C}$ et $\lambda=42$, (8) W/m.K.

Nous avons mesuré ce matériau par une méthode classique (montage symétrique avec isolation latérale des échantillons) et trouvé $44 \mathrm{~W} / \mathrm{m}$. K.

2.3 Première Conclusion. - Cette méthode testée sur un matériau isolant et sur un matériau conducteur s'avère aussi performante dans l'un et l'autre cas, ce qui lui donne un intérêt tout particulier. Nous avons utilisé un système de thermographie infrarouge, ce qui évite l'implantation de capteurs de température et

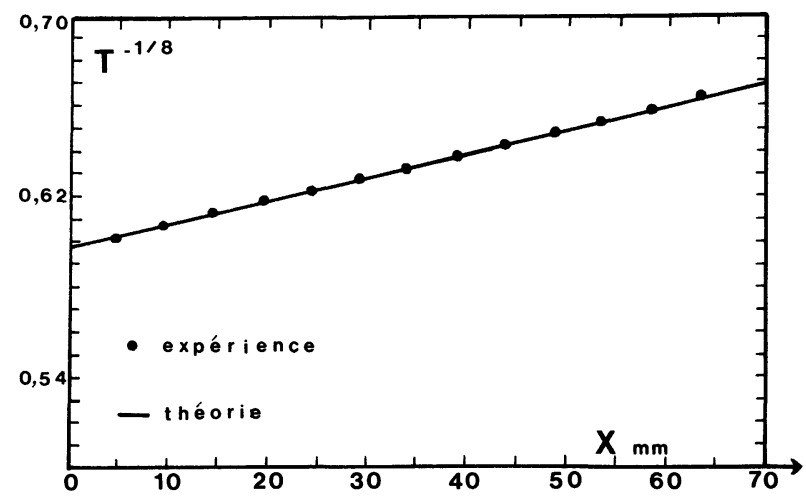

Fig. 5. - Thermogramme d'une plaque conductrice de la chaleur.

[Good conductor plate's thermogram.]

simplifie la méthode grâce à la visualisation du phénomène sur écran de télévision. Pour des matériaux relativement conducteurs (amortissement faible), nous pourrions utiliser des thermocouples, par exemple; par contre, pour des isolants, l'importance de l'amortissement (le phénomène s'étale sur $7 \mathrm{~mm}$ dans le cas du polyimide) ne permet pas d'envisager des thermocouples pour relever le champ de température.

2.4 DÉTERMINATION DE LA CONDUCTIVITÉ THERMIQUE LONGITUDINALE ÉQUIVALENTE D'UN BICOUCHE. - On envisage d'étudier un matériau composite simple constitué d'une couche isolante (bakélite) recouvert sur l'une de ses faces par un dépôt électrolytique de cuivre de quelques microns d'épaisseur.

Pris en série, la conductivité équivalente $\lambda_{\text {esérie }}$ aux deux couches est donnée par :

$$
\frac{e_{\mathrm{b}}+e_{\mathrm{c}}}{\lambda_{\text {esérie }}}=\frac{e_{\mathrm{b}}}{\lambda_{\mathrm{b}}}+\frac{e_{\mathrm{c}}}{\lambda_{\mathrm{c}}}
$$

où $e_{\mathrm{b}}$ et $e_{\mathrm{c}}$ sont les.épaisseurs de bakélite et de cuivre, $\lambda_{\mathrm{b}}$ et $\lambda_{\mathrm{c}}$ leurs conductivités respectives $\left(\lambda_{\mathrm{b}} \simeq 0,25 \mathrm{~W} /\right.$ $\left.\mathrm{m} . \mathrm{K} ; \lambda_{\mathrm{c}} \simeq 400 \mathrm{~W} / \mathrm{m} . \mathrm{K}\right)$.

$\lambda_{\mathrm{c}} \gg \lambda_{\mathrm{b}}$ et $e_{\mathrm{c}} \ll e_{\mathrm{b}}$ impliquent

$$
\lambda_{\text {esérie }} \simeq \lambda_{\mathrm{b}} \text {. }
$$

Pris en parallèle, la conductivité équivalente $\lambda_{\mathbf{e} \|}$ aux deux couches est donnée par :

$$
\lambda_{\mathrm{e} \|}=\frac{\lambda_{\mathrm{b}} e_{\mathrm{b}}+\lambda_{\mathrm{c}} e_{\mathrm{c}}}{e_{\mathrm{b}}+e_{\mathrm{c}}}
$$

Même pour des épaisseurs $e_{\mathrm{c}}$ très faibles (dépôt), le terme $\lambda_{\mathrm{c}} e_{\mathrm{c}}$ est souvent prépondérant devant le terme $\lambda_{\mathrm{b}} e_{\mathrm{b}}$, la conductivité $\lambda_{\mathrm{e} \|}$ ne peut être assimilée à celle de la bakélite $\left(\lambda_{\mathrm{e} \|} \neq \lambda_{\text {esérie }}\right)$. 
Le thermocoax est inséré dans la bakélite (Fig. 6). Dans la zone centrale, les faces apparentes de bakélite et de cuivre ont des températures très différentes (Fig. 7). Par contre, en s'éloignant de cette zone centrale, les profils de températures se rejoignent et l'hypothèse de barre devient vérifiée. En dépouillant dans cette zone, on est conduit pour $\varphi_{0}=18370 \mathrm{~W} / \mathrm{m}^{2}$ à :

$$
\begin{gathered}
K=768 \mathrm{~m}^{-2} \cdot \mathrm{K}^{-1 / 4} ; \quad T_{0}=55,9{ }^{\circ} \mathrm{C} \\
\lambda_{\mathrm{e} \|}=7,6 \text { (1) W/m.K. }
\end{gathered}
$$

On peut interpréter cette valeur comme correspondant à un dépôt de $28 \mu$ de cuivre.

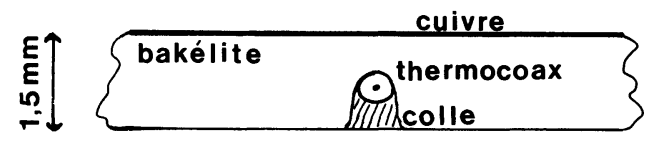

Fig. 6. - Schéma d'un bicouche.

[Bilayer's view.]

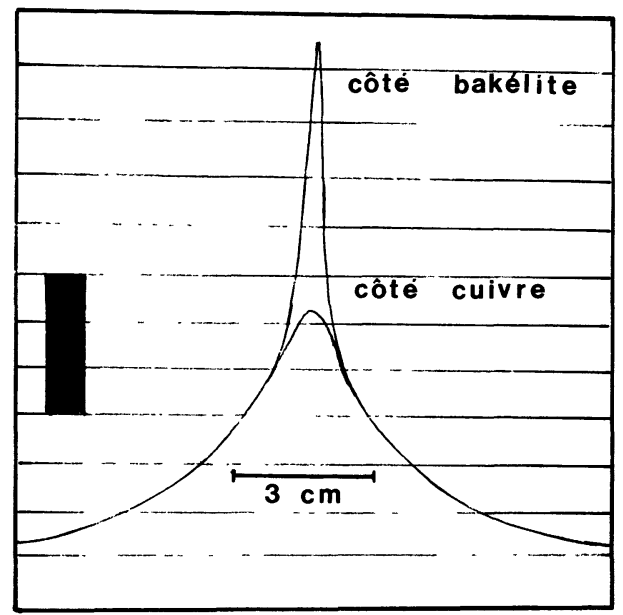

Fig. 7. - Visualisation de la thermographie d'un bicouche sur écran de télévision.

[Bilayer thermography view on a television set.]

\section{Conclusion.}

La méthode de détermination de la conductivité thermique longitudinale de plaques minces qui fait l'objet de cet article, complète bien les méthodes traditionnelles qui ne sont pas toujours adaptées à ce type de mesure. Elle apparaît aussi bien adaptée à des matériaux isolants que conducteurs, isotropes qu'anisotropes pourvu que les conditions d'échange superficiel permettent d'utiliser l'approximation de la "barre » $(B i<0,1)$. Sa mise en oeuvre est rendue simple par l'emploi d'un système performant de thermographie infrarouge. Le recoupement des résultats entre la méthode que nous présentons et des méthodes traditionnelles pour les matériaux isolant et conducteur étudiés est excellent; le coefficient de corrélation pour le tracé de la droite $T^{-1 / 8}=f(x)$ par régression linéaire est supérieur, pour tous les échantillons, à 0,99995. La précision obtenue sur la mesure de la conductivité thermique est donc équivalente à celle des méthodes traditionnelles soit de l'ordre de $3 \%$.

Cette méthode est appelée à connaître un devenir intéressant en thermique des systèmes électroniques où les substrats sont souvent livrés sous forme de plaques multicouches.

\section{Annexe.}

MESURE DE LA TEMPÉRATURE DE SURFACE D'UN CORPS À L'AIDE D'UNE CAMÉRA INFRAROUGE. - Le lecteur intéressé par la thermographie infrarouge pourra se reporter avec utilité à l'excellent livre de G. Gaussorgues " La thermographie infrarouge ".

Le flux de rayonnement thermique d'une surface ds est localisé sur un détecteur par l'intermédiaire d'une optique transparente dans la bande spectrale 8-12 $\mu \mathrm{m}$.

Ce détecteur transforme le signal thermique en signal électrique. Un système de balayage permet de voir les différents éléments d $s$ d'un objet et d'obtenir un signal vidéo visualisé sur écran de télévision.

La fonction de transfert globale résulte donc de la superposition des fonctions de transfert de l'optique, du détecteur, de l'électronique de traitement et de l'unité de visualisation.

Le flux thermique reçu par l'optique de la part de l'élément d $s$ à température $\theta$ placé dans un environnement à température uniforme $\theta_{\mathrm{a}}$ pour la bande spectrale $\lambda_{0}-\frac{\Delta \lambda}{2}, \lambda_{0}+\frac{\Delta \lambda}{2}$ est égal à

$$
\phi=\underbrace{\left[\int_{\lambda_{0}-\frac{\Delta \lambda}{2}}^{\lambda_{0}+\frac{\Delta \lambda}{2}} \varepsilon_{\lambda, \alpha} L_{\lambda, \theta}^{0} \mathrm{~d} \lambda\right.}_{\text {flux émis }}+\frac{\left.\int_{\lambda_{0}-\frac{\Delta \lambda}{2}}^{\lambda_{0}+\frac{\Delta \lambda}{2}}\left(1-\varepsilon_{\lambda, \alpha}\right) L_{\lambda, \theta_{\mathrm{a}}}^{0} \mathrm{~d} \lambda\right] \frac{\mathrm{d} s \cos \alpha}{r^{2}} S}{\text { flux réfléchi }}
$$

où $\varepsilon_{\lambda_{\alpha} \alpha}$ est l'émissivité spectrale pour la direction $\alpha$;

$L_{\lambda, \theta}^{0}$ la luminance spectrale du corps noir à la température $\theta$;

$r$ la distance de l'élément ds à l'optique de surface $S$. 
Nous limitant aux corps lambertiens $\left({ }^{4}\right)$ d'émissivité constante dans la bande spectrale considérée $\left(\varepsilon_{\lambda, \alpha}=\right.$ Cte $=\varepsilon)$

$$
\begin{gathered}
\phi \propto \varepsilon \int_{\lambda_{0}-\frac{\Delta \lambda}{2}}^{\lambda_{0}+\frac{\Delta \lambda}{2}} L_{\lambda, \theta}^{0} \mathrm{~d} \lambda+(1-\varepsilon) \int_{\lambda_{0}-\frac{\Delta \lambda}{2}}^{\lambda_{0}+\frac{\Delta \lambda}{2}} L_{\lambda, \theta_{\mathrm{a}}}^{0} \mathrm{~d} \lambda \\
\int_{\lambda_{0}-\frac{\Delta \lambda}{2}}^{\lambda_{0}+\frac{\Delta \lambda}{2}} L_{\lambda, \theta}^{0} \mathrm{~d} \lambda=\int_{\lambda_{0}-\frac{\Delta \lambda}{2}}^{\lambda_{0}+\frac{\Delta \lambda}{2}} \frac{2 h c^{2} \lambda^{-5}}{\exp \left(\frac{h c}{k \lambda \theta}\right)-1} \mathrm{~d} \lambda \\
=\underbrace{\frac{2 k^{4}}{h^{3} c^{2}}}_{A} \theta^{4} \underbrace{\frac{h c}{\mathrm{e}^{u}-1}}_{\frac{h c}{k \theta\left(\lambda_{0}+\frac{\Delta \lambda}{2}\right)}}=A \theta^{4} f(\theta)
\end{gathered}
$$

où $u=\frac{h c}{k \lambda \theta}$.

Il suit que

$$
\theta_{\mathrm{CN}}^{4} f\left(\theta_{\mathrm{CN}}\right)=\varepsilon \theta^{4} f(\theta)+(1-\varepsilon) \theta_{\mathrm{a}}^{4} f\left(\theta_{\mathrm{a}}\right)
$$

où $\theta_{\mathrm{CN}}$ est la température corps noir de l'élément ds envoyant le même flux thermique sur l'optique.

Le tableau ci-dessous donne les valeurs de $f(\theta)$ pour la bande spectrale 8-12 $\mu \mathrm{m}$.

\begin{tabular}{|c|c|c|c|c|c|c|c|c|}
\hline $\begin{array}{c}\theta \\
\text { en } \mathrm{K}\end{array}$ & 280 & 290 & 300 & 310 & 320 & 330 & 340 & 350 \\
\hline$f(\theta)$ & 1,59 & 1,66 & 1,71 & 1,76 & 1,80 & 1,83 & 1,86 & 1,87 \\
\hline$\theta$ & 360 & 380 & 400 & 500 & 600 & 700 & 800 & \multicolumn{1}{|c}{} \\
\cline { 1 - 7 } & 1,89 & 1,89 & 1,88 & 1,67 & 1,38 & 1,11 & 0,89 &
\end{tabular}

Pour des états de surface de forte émissivité, les faibles évolutions de $f(\theta)$ prouvent que la formule approchée :

$$
\theta_{\mathrm{CN}} \simeq \varepsilon \theta_{\mathrm{ap}}^{4}+(1-\varepsilon) \theta_{\mathrm{a}}^{4} \text { est utilisable } .
$$

Le tableau ci-après représente pour $\varepsilon=0,915$ et $\theta_{\mathrm{a}}=290 \mathrm{~K}$ les écarts $\theta-\theta_{\mathrm{ap}}$ pour différentes valeurs de $\theta_{\mathrm{CN}}$

\begin{tabular}{|l|c|c|c|c|c|c|c|}
\hline$\theta_{\mathrm{CN}}(\mathrm{en} \mathrm{K})$ & 300 & 310 & 320 & 330 & 340 & 350 & 360 \\
\hline$\theta-\theta_{\text {ap }}$ & $<0,2$ & $<0,2$ & $<0,2$ & $<0,2$ & $<0,2$ & 0,19 & 0,26 \\
\hline$\theta_{\mathrm{CN}}$ & 380 & 400 & 500 & 600 & 700 & 800 & \multicolumn{1}{|c}{} \\
\hline$\theta-\theta_{\text {ap }}$ & 0,51 & 0,67 & 2,3 & 4,4 & 5,2 & 6,6 \\
\cline { 1 - 5 }
\end{tabular}

L'écart $\theta-\theta_{\mathrm{ap}}$ est faible par rapport à la sensibilité maximale de la caméra infrarouge $(0,2 \mathrm{~K}$ sur la gamme $10^{\circ}$ pleine échelle, $0,4 \mathrm{~K}$ pour la gamme $20^{\circ}, 1 \mathrm{~K}$ pour la gamme $\left.50^{\circ}, \ldots\right)$.

$\left({ }^{4}\right)$ En fait les mesures sont faites autour de l'incidence normale $(\alpha \simeq 0$ ) dans le cadre de l'approximation de Gauss.
Mesure pratique d'uñe température. - Les plaques sont recouvertes d'une peinture noire mate dont l'émissivité dans la bande spectrale 8-12 $\mu \mathrm{m}$ a été préalablement mesurée à l'aide d'un étalon à température uniforme connue et variable dans le domaine de température ambiante $-100^{\circ} \mathrm{C}$.

Le fait de trouver une valeur de $\varepsilon$ constante pour la 
précision des mesures dans le domaine de température, à partir de la formule

$$
\theta_{\mathrm{CN}}^{4}=\varepsilon \theta^{4}+(1-\varepsilon) \theta_{\mathrm{a}}^{4}
$$

montre que $\varepsilon$ ne dépend pas ou très peu de la température mais aussi que les fonctions de transfert de l'optique, du détecteur, de l'électronique de traitement et de l'unité de visualisation sont uniformes dans la bande spectrale de fonctionnement de la caméra infrarouge.
La transcription du profil de température en températures vraies se fait alors à partir de

$$
\theta=\left|\frac{\theta_{\mathrm{CN}}^{4}-(1-\varepsilon) \theta_{\mathrm{a}}^{4}}{\varepsilon}\right|^{1 / 4}
$$

En fait, par l'intermédiaire d'un corps noir, on compare les profils de température à un étalon de température connu recouvert de la même peinture et envoyant le même flux thermique sur le détecteur de la caméra. L'utilité du corps noir comme intermédiaire est sa faible inertie thermique comparée à l'étalon.

\section{Biblingraphie}

[1] TYE, Thermal conductivity (Academic Press, London).

[2] Saint-BlanQuet, C., Mise au point d'un dispositif de mesure de la conductivité thermique des solides, D.E.A. (1968).

[3] ALNET, R., Mesures in situ et non destructives des caractéristiques thermophysiques : études préliminaires, D.E.A. (1983).

[4] Gaussorgues, G., La thermographie infrarouge (Technique et Documentation Lavoisier). 